\title{
Intrinsic and environmental effects on the interference properties of a high- performance quantum dot single-photon source
}

Gerhardt, Stefan; Iles-Smith, Jake; McCutcheon, Dara; He, Yu-Ming; Unsleber, Sebastian; Betzold, Simon; Gregersen, Niels; Mørk, Jesper; Höfling, Sven; Schneider, Christian

Published in:

Physical Review B (Condensed Matter and Materials Physics)

Link to article, DOI:

10.1103/PhysRevB.97.195432

Publication date:

2018

Document Version

Publisher's PDF, also known as Version of record

Link back to DTU Orbit

Citation (APA):

Gerhardt, S., lles-Smith, J., McCutcheon, D., He, Y-M., Unsleber, S., Betzold, S., Gregersen, N., Mørk, J., Höfling, S., \& Schneider, C. (2018). Intrinsic and environmental effects on the interference properties of a highperformance quantum dot single-photon source. Physical Review B (Condensed Matter and Materials Physics), 97, [195432]. https://doi.org/10.1103/PhysRevB.97.195432

\section{General rights}

Copyright and moral rights for the publications made accessible in the public portal are retained by the authors and/or other copyright owners and it is a condition of accessing publications that users recognise and abide by the legal requirements associated with these rights.

- Users may download and print one copy of any publication from the public portal for the purpose of private study or research.

- You may not further distribute the material or use it for any profit-making activity or commercial gain

- You may freely distribute the URL identifying the publication in the public portal 


\title{
Intrinsic and environmental effects on the interference properties of a high-performance quantum dot single-photon source
}

\author{
Stefan Gerhardt, ${ }^{1}$ Jake Iles-Smith, ${ }^{2}$ Dara P. S. McCutcheon, ${ }^{3}$ Yu-Ming He, ${ }^{1,4}$ Sebastian Unsleber, ${ }^{1}$ Simon Betzold, ${ }^{1}$ \\ Niels Gregersen, ${ }^{2}$ Jesper Mørk, ${ }^{2}$ Sven Höfling, ${ }^{1,5}$ and Christian Schneider ${ }^{1}$ \\ ${ }^{1}$ Technische Physik and Wilhelm Conrad Röntgen Research Center for Complex Material Systems, Physikalisches Institut, \\ Universität Würzburg, Am Hubland, D-97074 Würzburg, Germany \\ ${ }^{2}$ Department of Photonics Engineering, Technical University of Denmark, Ørsteds Plads, 2800 Kgs. Lyngby, Denmark \\ ${ }^{3}$ Quantum Engineering Technology Labs, H. H. Wills Physics Laboratory and Department of Electrical and Electronic Engineering, \\ University of Bristol, Bristol BS8 1FD, United Kingdom \\ ${ }^{4}$ Hefei National Laboratory for Physical Sciences at the Microscale and Department of Modern Physics, and CAS Center for Excellence and \\ Synergetic Innovation Center in Quantum Information and Quantum Physics, University of Science and Technology of China, \\ Hefei, Anhui 230026, China \\ ${ }^{5}$ SUPA, School of Physics and Astronomy, University of St. Andrews, St. Andrews KY16 9SS, United Kingdom
}

(Received 26 July 2017; revised manuscript received 5 April 2018; published 18 May 2018)

\begin{abstract}
We report a joint experimental and theoretical study of the interference properties of a single-photon source based on a $\operatorname{In}(\mathrm{Ga})$ As quantum dot embedded in a quasiplanar GaAs microcavity. Using resonant laser excitation with a pulse separation of $2 \mathrm{~ns}$, we find near-perfect interference of the emitted photons, and a corresponding indistinguishability of $\mathcal{I}=\left(99.6_{-1.4}^{+0.4}\right) \%$. For larger pulse separations, quasiresonant excitation conditions, increasing pump power, or with increasing temperature, the interference contrast is progressively and notably reduced. We present a systematic study of the relevant dephasing mechanisms and explain our results in the framework of a microscopic model of our system. For strictly resonant excitation, we show that photon indistinguishability is independent of pump power, but strongly influenced by virtual phonon-assisted processes which are not evident in excitonic Rabi oscillations.
\end{abstract}

DOI: 10.1103/PhysRevB.97.195432

\section{INTRODUCTION}

A central requirement for the implementation of single photons in quantum communication, quantum networks, linear optical quantum computing, and quantum teleportation is their degree of indistinguishability [1-5]. To this end, cold atoms, single ions, isolated molecules, optically active defects in diamonds, silicon carbide, and layered materials have all been identified as competitive sources of nonclassical light [6-13]. In the solid state, to date, epitaxially grown selfassembled semiconductor quantum dots (QDs) have proven to be the most promising candidates for producing single photons with high single-photon purity combined with high quantum efficiency [14-18]. More recently, by embedding QDs in optical microcavities, the possibility to also generate highly indistinguishable photons has emerged [19-24].

The indistinguishability condition can only be met if the overlap of the single-photon wave packets in frequency, polarization, space, and time is sufficient. Ideally, a QD produces photons with Fourier limited wave packets, such that their temporal extension can be expressed as $T_{2}=2 T_{1}$, where $T_{1}$ is the exciton lifetime. In reality, since a QD is embedded in a typically nonideal host medium, one must combat dephasing channels such as phonon coupling, or spectral wandering induced by coupling to carriers. If this coupling acts on the QD exciton on a timescale that is smaller than or comparable to photon-emission events, the spectral width of the emitted wave packets is broadened according to $\frac{1}{T_{2}}=\frac{1}{2 T_{1}}+\gamma$, with the characteristic dephasing time $T_{2}^{*}=1 / \gamma$ [25]. Furthermore, if the timing of emission events has some stochastic uncertainty, this will also reduce the expected wave-packet overlap and lead to a reduced indistinguishability $[25,26]$.

Previous experimental and theoretical studies have identified that the dominant dephasing processes in QD systems, namely, spectral wandering and electron-phonon scattering, occur on two different timescales [26-30]. Spectral wandering induced by charge noise occurs on a nanosecond timescale, as it takes time for charge carriers to accumulate around the QD, and is the dominant dephasing mechanism when there is a long delay between excitation pulses. This means that its influence on the coherence properties of emitted photons can be heavily suppressed by choosing an appropriately short pulse separation [21,22,27,31,32]. Phonon-induced dephasing, on the other hand, has a characteristic timescale of picoseconds [33-37] and impacts the spectral properties of the photons in two principle ways. The first is the emergence of a broad phonon sideband, related to the relaxation of the vibrational lattice of the host material during photon emission [24,38,39]. The second is a broadening of the zero phonon line due to virtual phonon processes-here a phonon in the material scatters off the QD, driving a virtual transition to an excited electronic state, and leading to a random phase change of the emitted photon [27,40,41].

One of the main challenges in the design, engineering, and operation of QD single-photon sources lies in achieving the maximum reduction of these dephasing processes, 


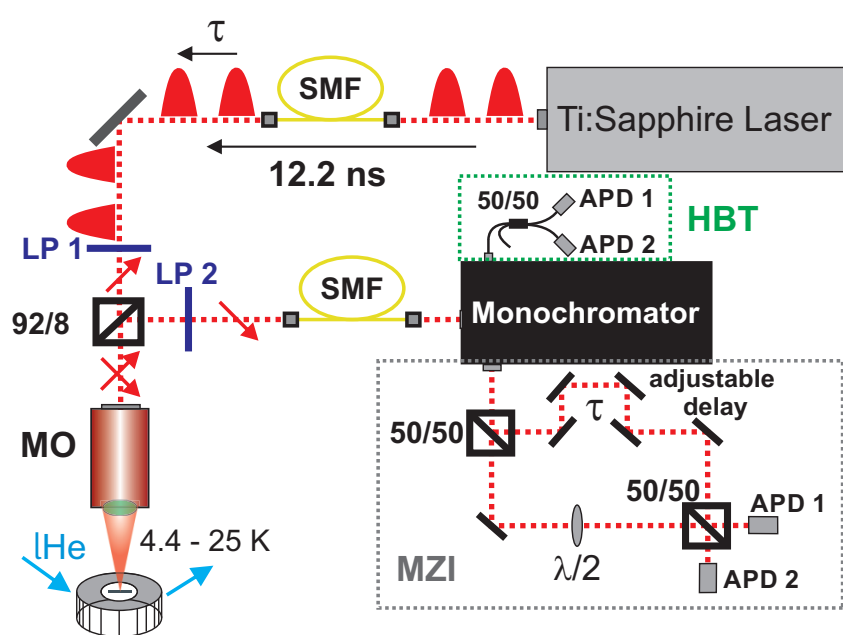

FIG. 1. Schematic of the experimental setup. The laser pulses separated by $12.2 \mathrm{~ns}$ are each divided into two pulses with variable pulse distance $\tau$. A cross-polarization configuration consisting of two linear polarizers (LP 1 and 2) suppresses the scattered laser light by a factor of $\approx 10^{7}$. After filtering by a single-mode fiber and a monochromator, the single photons are either directed to the HBT setup (green box) to measure the single-photon purity or, instead, to the unbalanced MZI (gray dotted box) to study photon indistinguishability.

which requires an accurate and precise understanding of their underlying microscopic origins. In this paper, we present a detailed study of emission from a single In(Ga)As QD which is embedded in a highly asymmetric, quasiplanar GaAs low- $Q$-factor microcavity. We demonstrate that while indistinguishable photons with almost ideal interference properties can be extracted from this source under resonant excitation conditions, the coherence is strongly affected by the pump configuration, the temperature, pump power, and the temporal separation between consecutive emission pulses. For strictly resonant excitation conditions, our main findings are that (a) virtual phonon transitions can strongly affect photon indistinguishability, though have little influence on excitonic Rabi oscillations, and (b) photon indistinguishability is independent of excitation pulse area. For quasiresonant excitation, we find noticeably lower indistinguishability values, which further decrease with increasing pump power. We analyze the experimental data using a model taking into account phonon processes that are both virtual and real in nature, spectral wandering due to charge noise, and timing jitter from delayed relaxation.

\section{EXPERIMENTAL SETUP AND SAMPLE DESCRIPTION}

A sketch of the implemented experimental setup is shown in Fig. 1. Short (full width at half maximum of $\delta t \approx 1.2 \mathrm{ps}$ ) coherent laser pulses with a repetition rate of $82 \mathrm{MHz}$ are generated by a Ti:sapphire laser, which are each then divided into two separate pulses with an adjustable delay $\tau$ by an unbalanced Mach-Zehnder interferometer. The spatial pulse shape is fine adjusted by a single-mode fiber (SMF) and the polarization is determined by a linear polarizer (LP 1). The laser signal is then coupled into the beam path via a $92 / 8$ pellicle beam splitter and focused on the QD sample by a microscope objective $(\mathrm{MO})(\mathrm{NA}=0.42)$. The QD sample is mounted on the cold finger of a lHe flow cryostat with tunable temperature. The microscope objective collects the emitted QD signal. The second linear polarizer (LP 2) is orientated perpendicular to the polarization of the laser, and suppresses the laser light scattered from the surface of the QD sample. Further filtering processes are accomplished by another SMF and by the monochromator with a grating up to $1500 \frac{\mathrm{lines}}{\mathrm{mm}}$, which is particularly necessary for resonant excitation. As well as suppressing stray-light from the excitation, this monochromator spectrally filters the QD signal itself, removing all emission outside a narrow $\sim 150 \mu \mathrm{eV}$ window.

By coupling the filtered QD signal onto a 50/50 beam splitter, we determine the second-order autocorrelation function of the source via a standard Hanbury, Brown, and Twiss (HBT) measurement. To measure the emitted photon indistinguishability, we use an asymmetric Mach-Zehnder interferometer (MZI) to perform a Hong-Ou-Mandel (HOM) interference measurement, where the two arms of the interferometer accurately settle the delay between two consecutively emitted photons. An additional $\lambda / 2$ wave plate can be inserted to rotate the polarization of the short arm by $90^{\circ}$ to make the two photons distinguishable. In both experiments, the photons are detected by two single-photon sensitive silicon based avalanche photodiodes at the exit ports of the second 50/50 beam splitter.

The source is composed of a low-density layer of $\operatorname{In}(\mathrm{Ga}) \mathrm{As}$ QDs embedded in an asymmetric planar cavity. The resonator consists of five (24) quarter-wavelength AlGaAs/GaAs mirror pairs in the top (bottom) distributed Bragg reflector and a 1$\lambda$-thick central cavity layer. High brightness of our source is ensured by oval defects which are self-aligned with the QDs and act as natural nanolenses, enabling a photon extraction efficiency exceeding $40 \%$ [42].

Figure 2(a) shows an above-band excitation spectrum of the planar sample with a resolution limited QD line at $\lambda_{\mathrm{QD}}=$ $933.6 \mathrm{~nm}$, which we associate with the neutral exciton transition. Although one can observe further emission features of neighboring QDs on this scale, the QD line of interest has no distracting emissions in the closest energetic vicinity. As we will detail later, in order to obtain a high photon indistinguishability, it is indispensable to deterministically generate highpurity single photons by the use of resonance fluorescence. The measured second-order autocorrelation function of the QD, which can be seen in Fig. 2(b), has been carried out under such resonant excitation conditions, with the delay between pulses set to their default value of $12.2 \mathrm{~ns}$. By fitting each pulse of the recorded coincidence histogram with a two-sided exponential decay convolved with a Gaussian distribution, we find $g^{(2)}(0)=0.006 \pm 0.002$. This result demonstrates highpurity single-photon emission of the considered QD. The QD lifetime obtained from these fittings is $T_{1} \approx 730 \mathrm{ps}$, which has also been confirmed by time-resolved photoluminescence (PL) measurements.

\section{RABI OSCILLATIONS AND PHONON COUPLING PARAMETERS}

Before we study the indistinguishability of the emitted photons, it is instructive to first investigate the power- and 

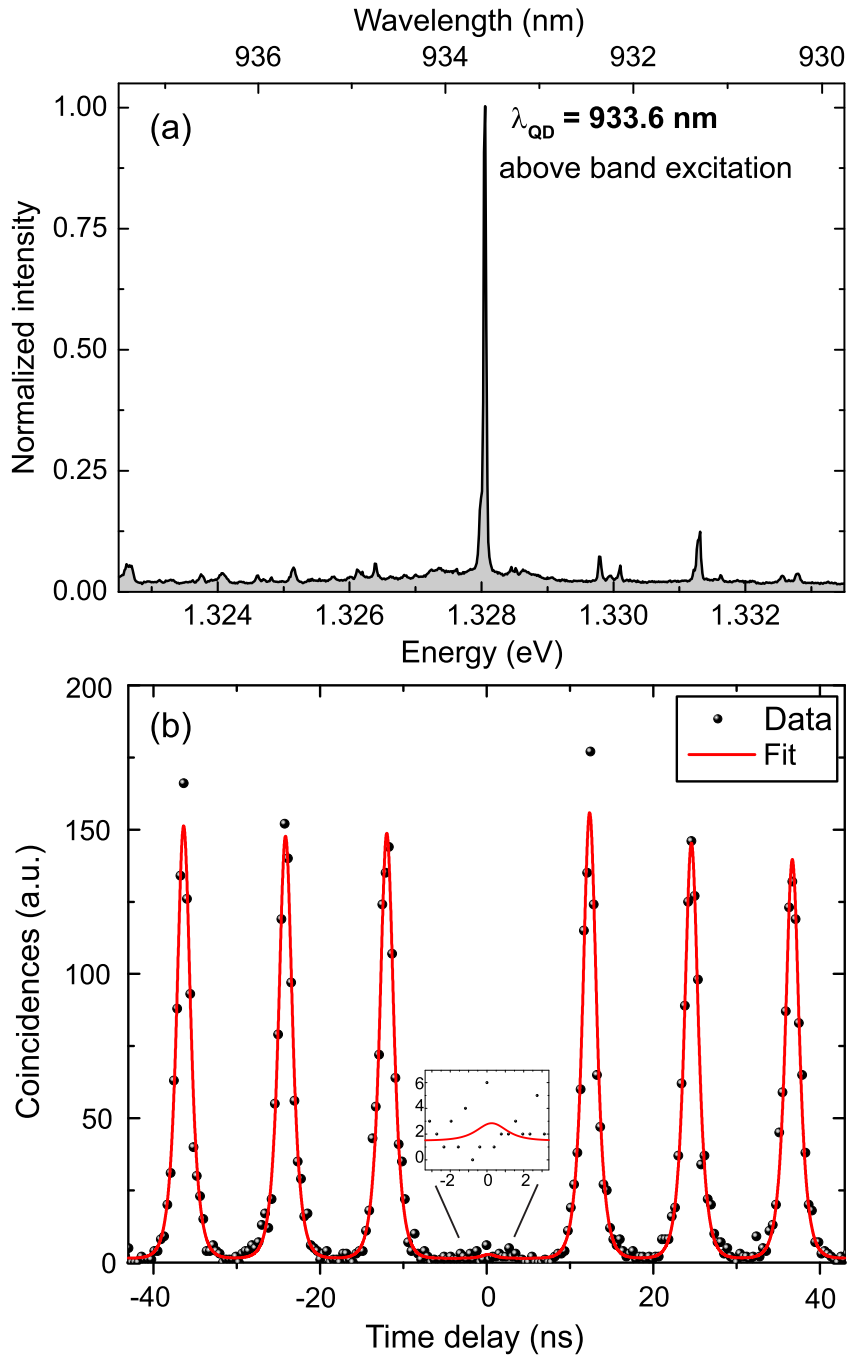

FIG. 2. (a) Spectrum of the investigated QD using above-band excitation. (b) Measured second-order coincidence histogram under resonant pulsed excitation conditions, with pulse separation $12.2 \mathrm{~ns}$ and a $\pi$-pump power of around $1.6 \mu \mathrm{W}$. The strong lack of coincidence counts around zero delay is an unambiguous signature of single-photon emission, and we extract $g^{(2)}(0)=0.006 \pm 0.002$.

temperature-dependent properties of our sample through excitonic Rabi oscillations. We excite the QD using resonant laser pulses with a fixed temporal width of $\delta t=1.2 \mathrm{ps}$ and a repetition rate of $82 \mathrm{MHz}$, and record the integrated intensity as a function of the square root of the laser power, and at various sample temperatures. The results are shown in Fig. 3 for temperatures of 5.6, 10, 15, 17.5, and $20 \mathrm{~K}$, where damped Rabi oscillations are clearly seen $[33,36,37,43,44]$. As the laser power is increased, a larger number of oscillations between the ground and single-exciton states can take place within the pulse duration. For increasing temperature, one can also see a clear decrease in overall intensity, as well as an increase in oscillation period.

In order to investigate these features further, we first fit our data to a simplified model of the form $c_{1}\left[1-\mathrm{e}^{-c_{2} A^{2}} \cos \left(c_{3} A\right)\right]$, where $c_{1}, c_{2}$, and $c_{3}$ are constants and $A$ denotes the pulse area, which due to our fixed pulse width satisfies $A \propto P_{\text {laser }}^{1 / 2}$
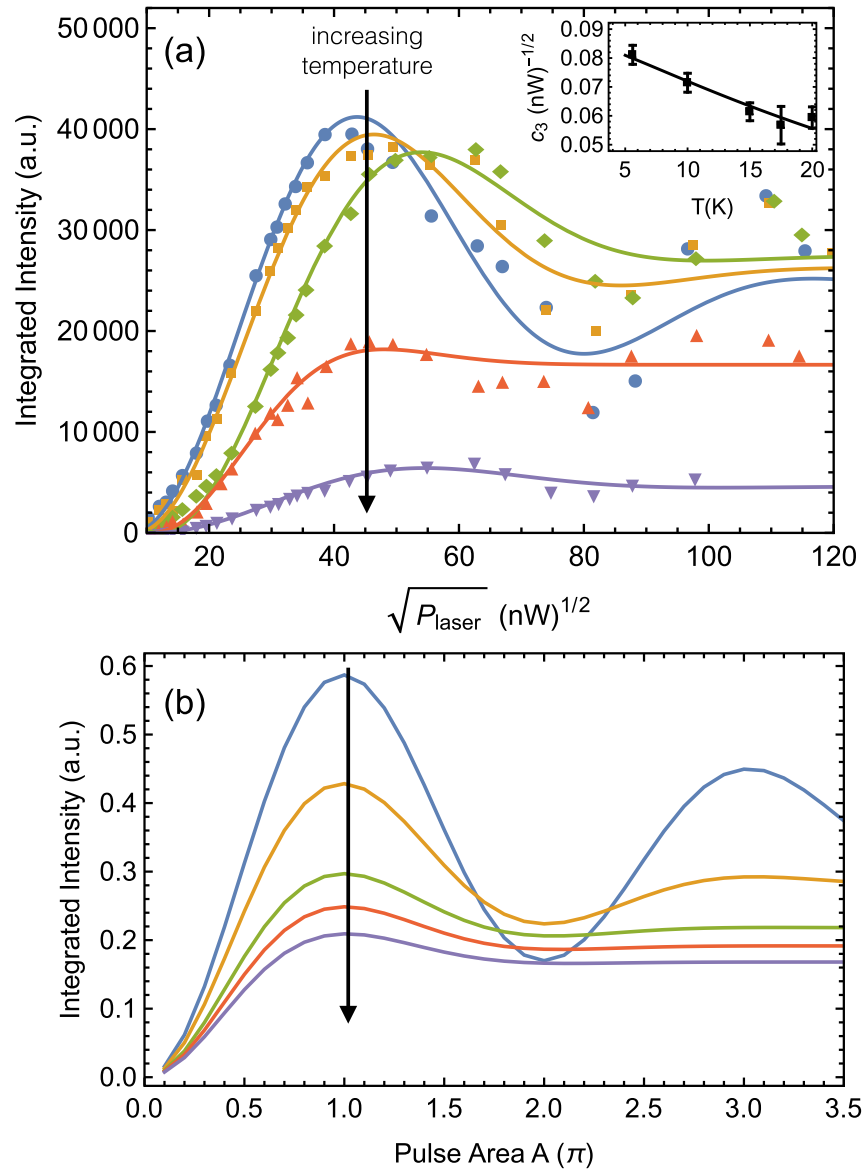

FIG. 3. (a) Rabi oscillations with increasing pulse power recorded for temperatures of $T=5.6,10,15,17.5,20 \mathrm{~K}$, ordered as indicated. The fits in (a) are to a pure-dephasing approximation to the phonon coupling theory. The variation of the oscillation period with temperature, shown by the plot markers in the inset, allows us to extract exciton-phonon coupling parameters, which lead to the solid curve in the inset. At higher temperatures, the maximum of the emitted intensity drops, and the $\pi$ pulse shifts towards more intense excitation power. (b) The result of a full phonon coupling model.

$[37,44]$. This form represents a pure-dephasing approximation and makes the simplification of temporally flat but finite pulses, such that the integrated intensity can be found from known expressions for the exciton excited-state population as a function of time [33], with the pulse area entering through the dependence on the Rabi frequency, $\Omega=A / \delta t$. Even within this simplified model, $A$ affects both the period of the oscillations and the damping through the quadratic dependence, which is a hallmark of excitation-induced dephasing $[33,36,43]$. We note, however, that in general coupling to phonons means the dependence of the Rabi oscillations on pulse power can be considerably more complex $[33,45]$. Broadly speaking, the pure-dephasing approximation is valid when $T_{1}^{-1} \ll \Omega \ll$ $k_{B} T$, with $\Omega \propto A$ the Rabi frequency. Within this approximation, the dominant phonon coupling effects are captured by a temperature-dependent Rabi frequency renormalization, here captured in the fitting constant $c_{3}$, and a dephasing rate which goes as the square of the Rabi frequency, hence the exponent in our expression. 


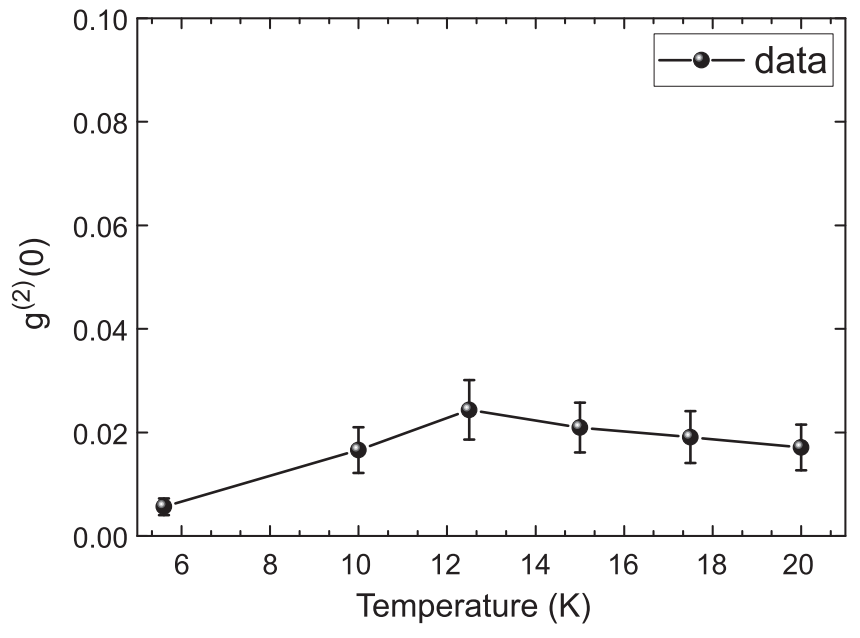

FIG. 4. Temperature-dependent $g^{(2)}(0)$ values, measured at $\pi$ pulse power each. A slight increase of the multiphoton probability from 0.006 to 0.024 is observable from 5.6 to $12.5 \mathrm{~K}$, whereas it marginally drops down to $0.017 \pm 0.004$ again at $20 \mathrm{~K}$.

The results of this fitting procedure are shown with the solid curves in Fig. 3(a), demonstrating generally good agreement. Despite the simplicity of the present model, we can use it to extract important exciton-phonon coupling parameters which we will use in our subsequent analysis. Specifically, within the pure-dephasing approximation that we use, real phonon transitions cause the Rabi frequency to be renormalized by the temperature-dependent Franck-Condon factor $B=$ $\exp \left\{-(1 / 2) \int_{0}^{\infty} d \omega J(\omega) \omega^{-2} \operatorname{coth}\left[\omega /\left(2 k_{B} T\right)\right]\right\}[33,39]$, which in our model is proportional to $c_{3}$. The renormalization factor contains the spectral density, which we take to be of the form $J(\omega)=\alpha \omega^{3} \exp \left[-\left(\omega / \omega_{c}\right)^{2}\right][36,44]$, with $\alpha$ an overall exciton-phonon coupling strength and $\omega_{c}$ a phonon cutoff frequency. From the trend of $c_{3}$ with temperature, shown in the inset of Fig. 3(a), we extract values of $\alpha=0.13 \pm 0.01 \mathrm{ps}^{-1}$ and $\omega_{c}=1.8 \pm 0.1 \mathrm{ps}^{-1}$. The values are comparable to those found previously $[35,36,44,46]$ and yield the solid curve shown in the inset.

The temperature-dependent $g^{(2)}(0)$ values each recorded at the particular $\pi$-pulse power are presented in Fig. 4. A slight increase of the multiphoton probability from $0.006 \pm 0.002$ to $0.024 \pm 0.006$ is observable from 5.6 to $12.5 \mathrm{~K}$, whereas it further marginally drops down to $0.017 \pm 0.004$ at $20 \mathrm{~K}$. The slightly increased value at $12.5 \mathrm{~K}$ might be induced by an intensified $\pi$-pulse excitation power, which is nearly doubled in comparison to the $5.6 \mathrm{~K}$ measurement. Except for the distinct lower value of $g^{(2)}(0)$ at $5.6 \mathrm{~K}$, altogether our measurements imply that the multiphoton probability is constant for temperatures up to $20 \mathrm{~K}$.

\section{Virtual phonon processes in Rabi oscillations}

Having partly characterized our system, it is interesting to consider whether Rabi oscillations can provide us with any additional characteristics pertinent to exciton-phonon coupling in our system. In particular, as previously mentioned, phonon interactions in QDs have been shown to lead to two primary mechanisms through which photons can lose coherence. The first is a coupling induced by displacement of the vibrational lattice due to the changes in the charge configuration of the QD $[24,38,39]$. During radiative recombination of an exciton, this can lead to the emission or absorption of a lattice excitation in addition to an emitted photon. This will produce a shift in the frequency of the emitted photon, leading to the emergence of a broad phonon sideband.

The second process is virtual in nature, in which an incoming phonon drives a virtual transition between the $s$ shell and higher-lying exciton states in the QD [40,47]. This is a pure-dephasing process where the scattering phonon induces a random phase change in the exciton, leading to broadening of the zero phonon line (ZPL). It is important to note that these two processes have very different temperature dependencies; real transitions occur for all temperatures as it is always possible to emit a phonon. Virtual phonon processes, however, require non-negligible phonon occupation, and therefore nonzero temperature, in order for a scattering event to occur. Thus, as was recently demonstrated by Reigue et al. [47], we expect real transitions to be the dominant dephasing mechanism at low temperatures, and virtual processes to contribute at higher temperatures $(T>10 \mathrm{~K})$.

In the appendices, we derive a master equation describing the laser-driven QD exciton, including both phonon coupling mechanisms described above. In Fig. 3(b), we show the results of the full phonon theory for the same temperatures as in Fig. 3(a). We see that the qualitative features are well reproduced, including an overall drop in intensity with temperature. This arises due to an ever greater fraction of phonons being emitted into the phonon sideband [24], which in these experiments is removed by filtering. Though the overall decrease in maximum intensity with increasing temperature is qualitatively captured by our model, we note that there are clear discrepancies. We believe these result from a variation of the sideband with temperature which is not quantitatively captured by our model, which assumes a spherically symmetric exciton wave function. We note that detailed study of the sideband dependence on temperature and QD shape would constitute an interesting and relevant study, though it is beyond the scope of this work, which instead seeks to explore the behavior of the ZPL under different driving and temperature conditions.

Interestingly, we find that the virtual phonon processes have little impact on Rabi oscillations; although these processes are included in Fig. 3(b), artificially removing them makes only imperceptible changes on the scale of the figure. This is because the damping of Rabi oscillations is dominated by strong driving-induced dephasing, making the real phonon processes orders of magnitude stronger than the virtual processes. Hence, although Rabi oscillations can be used to calibrate the real phonon-induced processes, they shed no light on the strength of virtual processes and the associated ZPL broadening which strongly affects indistinguishability, as we now explore in more detail.

\section{PHOTON INDISTINGUISHABILITY}

To measure the indistinguishability of the emitted single photons, we split each excitation pulse into a pair of pulses with 2 ns separation, and then couple these photons into an unbalanced Mach-Zehnder interferometer. Since one arm of the 

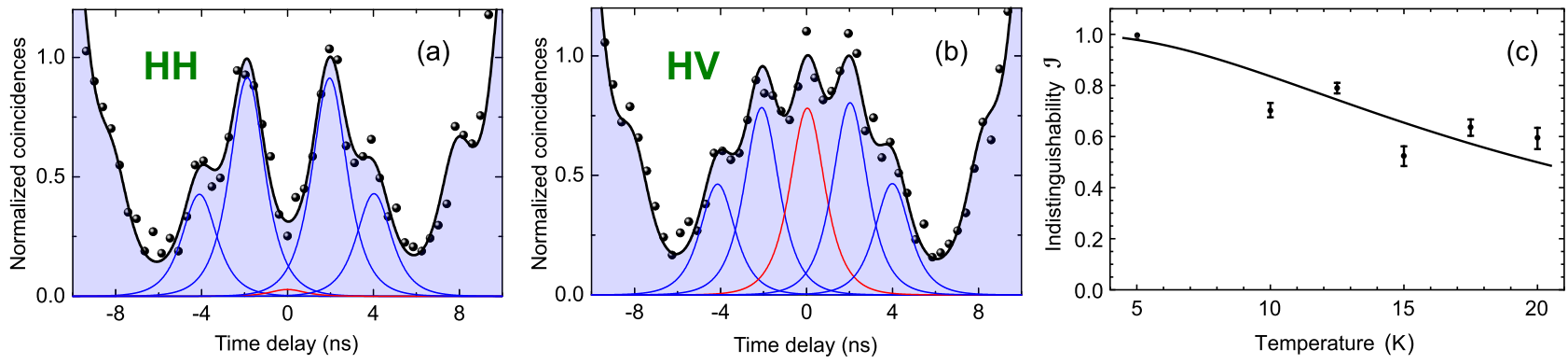

FIG. 5. Measured two-photon interference histograms for (a) parallel HH and (b) orthogonal photon polarization HV. The histograms are fitted with a sum of five two-sided exponential functions, each convolved with a Gaussian distribution. The measurements were carried out under $\pi$-pulse excitation with a pump power of $P_{\text {laser }}=(1.6 \pm 0.1) \mu \mathrm{W}$. These data lead to a corrected indistinguishability of $\mathcal{I}=\left(99.6_{-1.4}^{+0.4}\right) \%$. (c) Photon indistinguishability as a function of temperature (markers) and theoretical fit (solid line). Having spectrally removed the phonon sideband from the QD emission, the temperature dependence here arises due to zero-phonon-line broadening attributed to phonon-assisted virtual transitions.

interferometer is precisely adjusted to compensate for the delay between the two photons, the photons interfere at the second 50/50 beam splitter of the MZI, shown in Fig. 1. Figures 5(a) and 5(b) show the recorded coincidence histograms for parallel $(\mathrm{HH})$ and orthogonal polarization $(\mathrm{HV})$ of the photons. We see a near complete suppression of the central peak in Fig. 5(a), which is attributed to a high degree of indistinguishability of the emitted single photons. To quantitatively assess the degree of indistinguishability, we first calculate $v_{\text {raw }}=1-\left(A_{\mathrm{HH}} / A_{\mathrm{HV}}\right)$, which gives the raw visibility, defined in terms of the areas of the central peaks for parallel and perpendicular polarizations, and yielding a value of $v_{\text {raw }}=\left(96.3_{-1.4}^{+0.4}\right) \%$. Accounting for the measured $g^{(2)}(0)$ value, which slightly deviates from zero $\left[g^{(2)}(0)=0.006 \pm 0.002\right]$, and further taking into account the imperfections of the $50 / 50$ beam splitter as well as the nonunity contrast of the MZI $(1-\epsilon=0.99)$, we can correct the raw visibility to determine the true single-photon indistinguishability [16]. In doing so, we find a corrected indistinguishability of $\mathcal{I}=\left(99.6_{-1.4}^{+0.4}\right) \%[20,48]$, taken at $T=5.6 \mathrm{~K}$ and using strictly resonant $\pi$ pulses.

\section{A. Dependence on temperature}

We now explore the photon indistinguishability as described above as a function of sample temperature. As seen by the measured data (markers) in Fig. 5(c), as temperature is increased, photon indistinguishability correspondingly decreases. We recall that the monochromator used in our experiment spectrally filters the QD emission except for a narrow $\sim 150 \mu \mathrm{eV}$ window around the zero phonon line, meaning that the phonon sideband (arising from real phonon transitions and spread over $\sim \mathrm{meV}$ ) is almost entirely removed [24]. As such, the observed decrease in indistinguishability must arise from ZPL broadening.

We can therefore conclude that in contrast to the Rabi oscillations discussed above, virtual phonon processes do become important for indistinguishability measurements as temperature is increased. The reason for this difference is related to the different timescales being probed in the two types of measurement. For Rabi oscillations induced by temporally short ( $\sim 1 \mathrm{ps}$ ) pulses, only strong dephasing processes can have a significant impact. Virtual processes, having typical rates of $\sim 10^{-4} \mathrm{ps}^{-1}$ at $T=10 \mathrm{~K}$ [47] are too weak to affect Rabi oscillations, and instead the observed damping is dominated by real phonon processes, which take place on a timescale of $\pi \alpha k_{B} T(B \Omega)^{2} \approx 1 \mathrm{ps}^{-1}$ at the same temperature $[35,37,44]$ [for pulse areas of approximately $\pi$ with picosecond pulses such that $\Omega \approx \pi /(1 \mathrm{ps})]$.

Indistinguishability measurements, on the other hand, characterise emitted photon coherence following QD excitation and are therefore sensitive to processes comparable to the photonemission rate. In the present case, $\Gamma=T_{1}^{-1} \sim 10^{-3} \mathrm{ps}^{-1}$, allowing relatively weak virtual phonon processes to have an impact, as is seen in Fig. 5(c). This is confirmed by our theoretical model, as shown by the solid curve in the plot. Having removed the phonon sideband from the QD emission $[24]$ and for very short pulse separations $\left(\tau_{D} \leqslant 2 \mathrm{~ns}\right.$, as satisfied here), the photon coherence and hence the indistinguishability depend only on the emission rate $\Gamma$ and the phonon-induced dephasing rate $\gamma_{\mathrm{pd}}$. As shown in the appendices, we use the expression

$$
\mathcal{I}=\frac{\Gamma}{\Gamma+2 \gamma_{\mathrm{pd}}},
$$

with the temperature-dependent rate given by $[47,49]$

$$
\gamma_{\mathrm{pd}}=\frac{\alpha^{2} \mu}{\omega_{c}^{4}} \int_{0}^{\infty} \omega^{10} e^{-2\left(\omega / \omega_{c}\right)^{2}} n(\omega)[n(\omega)+1] d \omega,
$$

which is a microscopically derived extension of the phenomenological expression used in Ref. [27]. Three phonon coupling parameters enter these expressions. Using the values of $\alpha$ and $\omega_{c}$ already determined from the Rabi oscillations in Fig. 3, we fit the indistinguishability data to find the final parameter $\mu=1.1 \times 10^{-3} \mathrm{ps}^{2}$, which is related to the inverse level spacing between $s$ and $p$ states of the QD exciton. The result of this fitting procedure is shown by the solid curve in Fig. 5(c).

\section{B. Dependence on excitation conditions}

We now investigate the influence of the excitation conditions on the photon indistinguishability by varying the laser power. Furthermore, and in contrast to previous studies [31], this is done for strictly resonant $s$-shell excitation, as well as quasiresonant excitation with a QD-laser detuning of $34 \mathrm{meV}$, which is consistent with a $p$-shell resonance of the QD $[26,50]$. The indistinguishability values were then extracted for $50 \%$, 

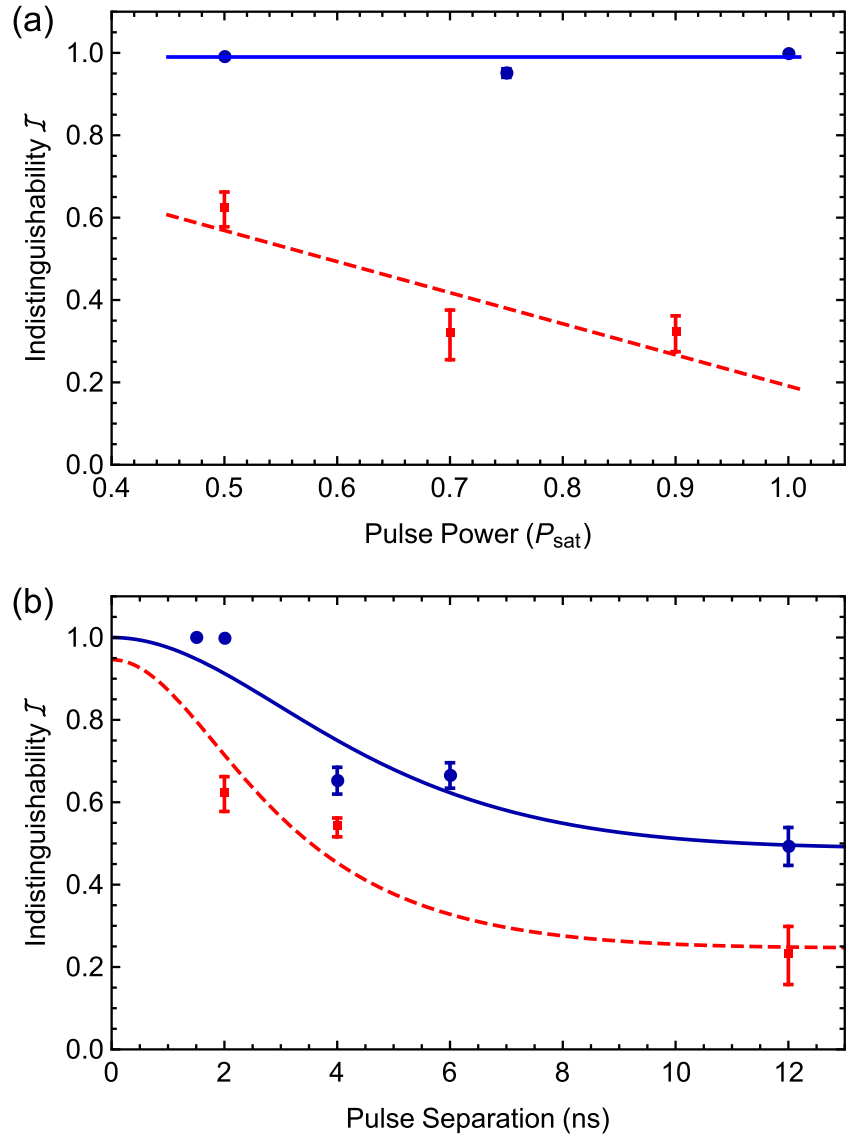

FIG. 6. (a) Indistinguishability as a function of excitation power. For resonant $s$-shell excitation (blue circles), the $x$ axis corresponds to the pump power as a fraction of a $\pi$ pulse. For quasiresonant $p$ shell excitation (red squares), we excite $\approx 34 \mathrm{meV}$ above the $s$ shell, with the $x$ axis now corresponding to the fraction with respect to the saturation power. (b) Indistinguishability as a function of the pulse separation. The solid blue curve is a fit to a resonant excitation model assuming dephasing only caused by charge fluctuations with a finite correlation timescale of $\tau_{C} \sim 6 \mathrm{~ns}$. The dashed red curve corresponds to a theoretical model also including timing jitter. Both measurements, (a) and (b), were carried out at $5.6 \mathrm{~K}$.

$70 \%$, and $90 \%$ of the QD saturation level under $p$-shell excitation, and for intensities equivalent to $50 \%, 75 \%$, and $100 \%$ with respect to the maximum of the Rabi oscillation in Fig. 3(a) for the resonant exciton.

The results are presented in Fig. 6(a). Comparing the $s$ - and $p$-shell data sets, for the latter we can immediately see the important role of uncertainties in the relaxation time from the $p$ to $s$ shell (timing jitter), and also the increased probability of exciting charge carriers in the vicinity of the QD (giving rise to dephasing), both of which suppress indistinguishability $[25,26]$. Additionally, compared to the resonant pumping conditions, which give a near-unity degree of indistinguishability for all chosen pump powers, the indistinguishability for $p$-shell excitation significantly drops down from $\mathcal{I}=(62 \pm 4) \%$ for $P=0.5 P_{\text {sat }}$ to $\mathcal{I}=(32 \pm 4) \%$ for $P \approx 0.9 P_{\text {sat }}$.

Timing jitter is parameterized by a single decay constant characterizing the $p$ - to $s$-shell transition, which, being phonon mediated, is expected to be independent of optical excita- tion power [50]. The decrease of indistinguishability with increasing power, as has been observed elsewhere [31], gives us an indication as to the relative strength of timing jitter and dephasing caused by charge fluctuations. Although a complete set of power-dependent measurements (including very low powers) would be necessary to fully determine the relative strengths of these two mechanisms, we can nevertheless assume that the dephasing contribution leads to a linear decrease in indistinguishability with power, as was found in Ref. [31]. The result of this fitting procedure is shown by the dashed red curve and, by extrapolating to zero power, we find $\mathcal{I} \rightarrow 95 \%$. Within these assumptions, at zero power, the value of $\mathcal{I}$ is now dominated by timing jitter and given by $\mathcal{I}=\Gamma_{p \rightarrow s} /\left(\Gamma_{p \rightarrow s}+\Gamma\right)$, where $\Gamma_{p \rightarrow s}^{-1}=53$ ps is the $p$ - to $s$-shell relaxation time.

Turning now to strictly resonant excitation, interestingly there is little change in the measured indistinguishability as a function of power. This suggests that the power-dependent dephasing processes present for $p$-shell excitation are now suppressed, and also that the power-dependent dephasing observed in the Rabi oscillations in Fig. 3 does not affect photon indistinguishability. The apparent absence of any power-dependent dephasing can be explained by noting that the saturation power for $s$-shell excitation is significantly smaller than for $p$-shell excitation, and one may therefore expect the creation of charge carriers to be correspondingly suppressed. Additionally, the insensitivity of indistinguishability measurements to the phonon-induced damping seen in Fig. 3 can be explained by noting that phonons lead to thermalization in the dressed-state basis, mediating population transfer between the system eigenstates, and giving rise to a reduced population in the single-exciton state. Measurements of indistinguishability, however, are normalized in such a way as to naturally postselect events where the QD has been successfully excited, removing any dependence on the initial QD population. More generally, as we show in the appendices, for very short excitation pulses satisfying $\delta t / T_{1} \ll 1$, indistinguishability is independent of the QD state immediately following excitation.

\section{Dependence on pulse separation}

While phonons affect the coherence of QD excitons on a timescale which is short compared to the exciton lifetime, the effects of fluctuating charges and spin noise occur on a nanosecond timescale [27]. Thus, in order to quantify the impact of these slower channels on the photon interference, it is instructive to study the indistinguishability as a function of the pulse separation between the arriving excitation pulses. To do so, we vary the pulse separation between 2 and $12 \mathrm{~ns}$, which maps out the characteristic correlation time of dephasing processes which act on the source. This is repeated for resonant ( $\pi$-pulse conditions) as well as quasiresonant excitation conditions ( $50 \%$ of the saturation level).

The results are plotted in Fig. 6(b), where in both cases we observe a drop in the indistinguishability as the pulse separation is increased. In order to gain a quantitative understanding, we make use of the expression developed by Thoma et al. [27], for which charge and spin noise are assumed to lead to a stochastic fluctuation of the excited-state energy level, and taken to have Gaussian statistics. By taking an ensemble 
average at a given pulse separation $\tau_{D}$ over the noise correlation function, a Gaussian form for the associated dephasing rate can be obtained as

$$
\tilde{\gamma}\left(\tau_{D}\right)=\tilde{\gamma}_{0}\left(1-e^{-\left(\tau_{D} / \tau_{C}\right)^{2}}\right),
$$

where $\tau_{C}$ quantifies the finite correlation time of the environment and $\tilde{\gamma}_{0}$ parameterizes the overall strength of fluctuations. In general, this rate should be added to the temperaturedependent contribution in Eq. (2), though in the following this latter contribution is ignored due to the low temperature of $T=$ $5.6 \mathrm{~K}$ used. We therefore use simply $\mathcal{I}=\Gamma /\left[\Gamma+2 \tilde{\gamma}\left(\tau_{D}\right)\right]$ to fit the $s$-shell excitation data in Fig. 6(b), with the results shown by the solid blue curve. The fitting parameters we find are $\tilde{\gamma}_{0}=$ $0.37 \mu \mathrm{eV}$ and $\tau_{C}=6.48 \mathrm{~ns}$, which give $\mathcal{I}=0.49$ as $\tau_{D} \rightarrow \infty$.

In the $p$-shell excitation case, the situation is somewhat more complicated. Even in the absence of any phonon-induced dephasing, we expect both timing jitter and a power-dependent dephasing rate to reduce phonon indistinguishability $[25,26]$. We can, nevertheless, use the $p$ - to $s$-shell relaxation rate $\Gamma_{p \rightarrow s}$ found from Fig. 6(a), and again assume that all the dephasing mechanisms causing a loss in indistinguishability are described by Eq. (3). Including both timing jitter and dephasing, we fit the data in Fig. 6(b) to

$$
\mathcal{I}=\left(\frac{\Gamma_{p \rightarrow s}}{\Gamma_{p \rightarrow s}+\Gamma}\right)\left(\frac{\Gamma}{\Gamma+2 \tilde{\gamma}\left(\tau_{D}\right)}\right),
$$

with the result shown by the dashed red curve, and we find fitting parameters $\tilde{\gamma}_{0}=1.0 \mu \mathrm{eV}$ and $\tau_{C}=5.8 \mathrm{~ns}$. As is expected from Fig. 6(a), $s$ - and $p$-shell excitation conditions give rise to different dephasing rates $\tilde{\gamma}$. More intriguingly, by comparison of Figs. 6(a) and 6(b), it appears this dephasing process behaves similarly as a function of time (pulse separation), but quite differently as a function of power. Whether the dephasing taking place in each case is of the same origin remains to be seen, and it would be interesting to investigate the entire pumppower-pulse-separation parameter space in future studies.

\section{CONCLUSION}

We have studied both Rabi oscillations and photon indistinguishability for a $\mathrm{QD}$ in a quasiplanar low- $Q$ microcavity. For resonant excitation of a single exciton, we found that increasing temperatures strongly affected indistinguishability, though it had little influence on excitonic Rabi oscillations. With increasing pump power, we found that indistinguishability was unaffected in the resonant case, though it was strongly reduced for quasiresonant excitation conditions even for short pulse separations, suggesting a fast dephasing process acting on a timescale shorter than 2 ns. With increasing pulse separation, indistinguishability dropped in both the resonant and quasiresonant cases, from which we deduce a characteristic noise correlation time of approximately $6 \mathrm{~ns}$.

\section{ACKNOWLEDGMENTS}

We acknowledge support by the State of Bavaria and the German Ministry of Education and Research (BMBF) within the project Q.com. J.I.-S. and J.M. acknowledge support from the Danish Research Council (Grant No. DFF-4181-00416) and Villum Fonden (NATEC Centre). This project has received funding from the European Union's Horizon 2020 research and innovation programme under the Marie Skłodowska-Curie Grant Agreement No. 703193.

\section{APPENDIX A: EXCITON-PHONON COUPLING MODEL}

We model the QD as a two-level system with ground state $|0\rangle$ and first exciton state $|X\rangle$, corresponding to the $s$-shell transition of the QD with energy $\omega_{X}$. The excitation is consider to be a semiclassical laser pulse with carrier frequency $\omega_{l}$, and a Gaussian envelope function $\Omega(t)=$ $(A / 2 \delta \tau \sqrt{\pi}) \exp \left[-\left(t-t_{0}\right)^{2} /(2 \delta \tau)^{2}\right]$, where $A$ is the pulse area and $\delta \tau$ is the temporal pulse width, such that $\delta \tau=\delta t /(4 \sqrt{\ln 2})$ with $\delta t$ the full width at half maximum. By moving to a frame rotating with respect to the carrier frequency and making the rotating wave approximation, we obtain a time-dependent system Hamiltonian $H_{S}(t)=\frac{\delta}{2} \sigma_{z}+\frac{\Omega(t)}{2} \sigma_{x}$, where we have defined the detuning $\delta=\omega_{X}-\omega_{L}$. The Pauli operators take their standard form $\sigma_{x}=|0\rangle\langle X|+| X\rangle\langle 0|, \sigma_{y}=i(|0\rangle\langle X|-$ $|X\rangle\langle 0|)$, and $\sigma_{z}=|X\rangle\langle X|-| 0\rangle\langle 0|$.

To describe the influence of phonon dephasing mechanisms, we model the phonon environment as a collection of harmonic oscillators with free Hamiltonian $H_{B}=\sum_{k} v_{k} b_{k}^{\dagger} b_{k}$, where $b_{k}$ is the annihilation operator for the $\boldsymbol{k}$ th mode of the phonon environment with frequency $v_{k}$. The electron-phonon interaction is then governed by the Hamiltonian $H_{I}=|X\rangle\langle X|\left(V_{L}+V_{Q}\right)$. The first term, $V_{L}=\sum_{k} g_{k}\left(b_{k}^{\dagger}+b_{k}\right)$, leads to real phonon dephasing mechanisms as well as the emergence of the phonon sideband [24,39,47]. The linear electron-phonon coupling strength is given by the matrix elements $g_{\boldsymbol{k}}=\sum_{a=e, h} M_{a, \boldsymbol{k}}^{11}$ for electrons $(e)$ and holes $(h)$, where for deformation potential coupling we have

$$
M_{a, \boldsymbol{k}}^{i j}=\sqrt{\frac{\nu_{\boldsymbol{k}}}{2 \varrho c_{s}^{2} \mathcal{V}}} D_{a} \int d^{3} r \psi_{i a}^{*}(\boldsymbol{r}) \psi_{j a}(\boldsymbol{r}) e^{i \boldsymbol{k} \cdot \boldsymbol{r}},
$$

which is the matrix element corresponding to the phononinduced transition between the $i$ th and $j$ th electronic states. Here, $\varrho$ is the mass density, $c_{s}$ is the speed of sound in the material, and $\mathcal{V}$ is the phonon normalization volume. This matrix element depends on the wave function $\psi_{i, e / h}(\boldsymbol{r})$ of the confined electron/hole and the corresponding deformation potential $D_{a}$.

As introduced by Muljarov and Zimmerman [40], virtual phonon-assisted processes can be described by a quadratic interaction term $V_{Q}=\sum_{\boldsymbol{k}, \boldsymbol{k}^{\prime}} f_{\boldsymbol{k}, \boldsymbol{k}^{\prime}}\left(b_{\boldsymbol{k}}+b_{\boldsymbol{k}}^{\dagger}\right)\left(b_{\boldsymbol{k}^{\prime}}+b_{\boldsymbol{k}^{\prime}}^{\dagger}\right)$, where higher-lying states are eliminated perturbatively, allowing us to treat the QD as a two-level system. The effective coupling for the virtual processes is given by $f_{\boldsymbol{k}, \boldsymbol{k}^{\prime}}=$ $\sum_{a=e, h} \sum_{j>1} M_{a, \boldsymbol{k}}^{1 j} M_{a, \boldsymbol{k}^{\prime}}^{j 1}\left[\omega_{m}^{a}-\omega_{1}^{a}\right]^{-1}$, where $\omega_{m}^{e / h}$ is the energy of the $m$ th electron/hole energy level.

\section{APPENDIX B: RABI OSCILLATION MASTER EQUATION}

To describe the effect of phonon interactions on the exciton dynamics, we shall use a polaron master-equation approach $[33,39,47,51]$. Here we apply the unitary transformation $\mathcal{U}_{V}=\exp \left(-\sigma^{\dagger} \sigma \otimes S\right)$, with $S=\sum_{k} v_{k}^{-1} g_{k}\left(b_{k}^{\dagger}-b_{k}\right)$, to the electron-phonon coupling Hamiltonian defined above. This leads to a displaced representation of the phonon environment, providing an optimized basis for a perturbative description of the QD dynamics. Importantly, this transformation naturally 
captures the non-Markovian relaxation behavior of the phonon environment during exciton recombination [24,39,47].

Applying the polaron transformation to the Hamiltonian yields $H_{V}=\mathcal{U}_{V}^{\dagger} H \mathcal{U}_{V}=H_{S}+H_{I}+H_{B}$, where

$$
\begin{aligned}
& H_{S}=\frac{\tilde{\delta}}{2} \sigma_{z}+\frac{\Omega_{r}(t)}{2} \sigma_{x}, H_{B}=\sum_{\boldsymbol{k}} v_{\boldsymbol{k}} b_{k}^{\dagger} b_{\boldsymbol{k}}, \\
& H_{I}=\frac{\Omega(t)}{2}\left(\sigma_{x} B_{x}+\sigma_{y} B_{y}\right)+|X\rangle\langle X| V_{Q} .
\end{aligned}
$$

Here, $\tilde{\delta}=\delta+\sum_{k} v_{k}^{-1} g_{k}^{2}$ is the polaron-shifted detuning, and the driving term $\Omega_{r}(t)=B \Omega(t)$ has been renormalized by the thermal expectation of the lattice displacement operator $B=\operatorname{tr}\left[\exp ( \pm S) \rho_{B}\right]$ and $\rho_{B}=\exp \left(-\beta H_{B}\right) / \operatorname{tr}\left[\exp \left(-\beta H_{B}\right)\right]$, with the thermodynamic temperature defined as $\beta=1 / k_{B} T$. In this transformed representation, the system now couples to the phonon environment through the displacement operators $B_{x}=\left(B_{+}+B_{-}-2 B^{2}\right) / 2$ and $B_{y}=i\left(B_{+}-B_{-}\right) / 2$, with $B_{ \pm}=\exp ( \pm S)$.

This Hamiltonian allows us to derive a second-order master equation in the polaron frame which captures multiphonon processes [33]. Using the Born-Markov approximation [52], we may write the Schrödinger picture master equation as

$$
\begin{aligned}
\frac{\partial \rho(t)}{\partial t}= & -i\left[\frac{\delta}{2} \sigma_{z}+\frac{\Omega_{r}(t)}{2} \sigma_{x}, \rho(t)\right]+\int_{0}^{\infty} d \tau\left\{\left[\sigma^{\dagger} \sigma, \sigma^{\dagger} \sigma(t-\tau, t) \rho(t)\right] \Lambda_{z}(\tau)+\text { H.c. }\right\} \\
& +\frac{\Omega(t)}{4} \int_{0}^{\infty} d \tau \Omega(t-\tau)\left\{\left[\sigma_{x}, \sigma_{x}(t-\tau, t) \rho(t)\right] \Lambda_{x}(\tau)+\left[\sigma_{y}, \sigma_{y}(t-\tau, t) \rho(t)\right] \Lambda_{y}(\tau)+\text { H.c. }\right\}
\end{aligned}
$$

Here we have defined $\sigma_{i}(s, t)=U_{0}(t) U_{0}^{\dagger}(s) \sigma_{i} U_{0}(s) U_{0}^{\dagger}(t)$, where $U_{0}(t)=\exp \left[-i \int_{0}^{t} H_{S}(s) d s\right]$ is the interaction picture transformation. The polaronic correlation functions take the standard form [33],

$$
\begin{aligned}
& \Lambda_{x}(\tau)=\frac{B^{2}}{2}\left(e^{\varphi(\tau)}+e^{-\varphi(\tau)}-2\right), \\
& \Lambda_{y}(\tau)=\frac{B^{2}}{2}\left(e^{\varphi(\tau)}-e^{-\varphi(\tau)}\right),
\end{aligned}
$$

where we have defined the phonon propagator as $\varphi(\tau)=\int_{0}^{\infty} d \nu \nu^{-2} J(\nu)\left[\cos \nu \tau \operatorname{coth}(\beta \nu / 2)-i \sin \nu_{k} \tau\right]$

Here the spectral density takes the standard form $J(v)=$ $\alpha v^{3} \exp \left(-v^{2} / v_{c}^{2}\right)$, where $\alpha$ is the linear electron-phonon coupling strength, set by the deformation potential, and $v_{c}$ is the cutoff frequency, set by the size of the exciton wave function [44]. In addition to the polaronic dephasing terms, we also have a correlation function associated with the virtual transitions: $\Lambda_{z}(\tau)=\sum_{\boldsymbol{k}, \boldsymbol{k}^{\prime}} \sum_{\boldsymbol{q}, \boldsymbol{q}^{\prime}} f_{\boldsymbol{k}, \boldsymbol{k}^{\prime}} f_{\boldsymbol{q}, \boldsymbol{q}^{\prime}}\left\langle\left[b_{\boldsymbol{k}}(\tau)+\right.\right.$ $\left.\left.b_{\boldsymbol{k}}^{\dagger}(\tau)\right]\left[b_{\boldsymbol{k}^{\prime}}(\tau)+b_{\boldsymbol{k}^{\prime}}^{\dagger}(\tau)\right]\left(b_{\boldsymbol{q}}+b_{\boldsymbol{q}}^{\dagger}\right)\left(b_{\boldsymbol{q}^{\prime}}+b_{\boldsymbol{q}^{\prime}}^{\dagger}\right)\right\rangle$. For a QD with a spherically symmetric wave function, the primed and unprimed modes may be factorized-this is equivalent to assuming that the incoming and outgoing scattering modes are different [47]. Assuming that the dominant virtual transition is between the $s$ and $p$ shells, we find that

$$
\Lambda_{z}(\tau)=\left[\int_{0}^{\infty} d \omega \mathcal{J}(\omega)\left\{n(\omega) e^{i \omega \tau}+[n(\omega)+1] e^{-i \omega \tau}\right\}\right]^{2},
$$

where we have defined the thermal occupation as $n(\omega)=[\exp (\beta \omega)-1]^{-1}$. We have also introduced $\mathcal{J}(\omega)=\sqrt{\alpha}_{Q} \omega^{5} \exp \left(-\omega^{2} / \omega_{c}^{2}\right), \quad$ where $\quad \alpha_{Q}=\alpha^{2} \omega_{c}^{-4} \mu$ is the virtual phonon coupling strength. Here, $\mu=$ $\pi\left[D_{e}-D_{h}\right]^{-4}\left(D_{e}^{2} \Delta_{e}^{-1}+D_{h}^{2} \Delta_{h}^{-1}\right)$ and $\Delta_{e / h}$ is the splitting between the $s$ and $p$ shells for the electron/hole. A detailed derivation of this expression can be found in the supplement of Ref. [47].

In the Markov approximation, the phonon-correlation function decays on a timescale much faster than the system dynamics. This allows us to make an adiabatic approxima- tion when transforming to the interaction picture [33], such that $\sigma_{i}(t-\tau) \approx \exp \left[-i H_{s}(t) \tau\right] \sigma_{i} \exp \left[i H_{s}(t) \tau\right]$. On resonance with polaron-shifted transition energy, $\tilde{\delta}=0$, the interaction picture system operators then take the form

$$
\begin{aligned}
\sigma_{x}(t-\tau, t)= & \sigma_{x}, \\
\sigma_{y}(t-\tau, t)= & \sin \left[\Omega_{r}(t) \tau\right] \sigma_{z}+\cos \left[\Omega_{r}(t) \tau\right] \sigma_{y}, \\
\sigma^{\dagger} \sigma(t-\tau, t)= & \frac{1}{2}\left\{1-\cos \left[\Omega_{r}(t) \tau\right]\right\} \mathbb{1} \\
& +\cos \left[\Omega_{r}(t) \tau\right] \sigma_{z}+\frac{1}{2} \sin \left[\Omega_{r}(t) \tau\right] \sigma_{y} .
\end{aligned}
$$

Substituting these expressions into our master equation, we have $\dot{\rho}(t)=-\frac{i \Omega_{r}(t)}{2}\left[\sigma_{x}, \rho(t)\right]+\mathcal{K}_{L}[\rho(t)]+\mathcal{K}_{Q}[\rho(t)]$, where we have defined the superoperator associated to real phonon transitions as

$$
\begin{aligned}
\mathcal{K}_{L}[\rho(t)]= & -\left[\frac{\Omega(t)}{2}\right]^{2}\left\{\left[\sigma_{x}, \sigma_{x} \rho(t)\right] \Gamma_{1}(t)\right. \\
& \left.+\left[\sigma_{y}, \sigma_{y} \rho(t)\right] \Gamma_{2}(t)+\left[\sigma_{y}, \sigma_{z} \rho(t)\right] \Gamma_{3}(t)+\text { H.c. }\right\},
\end{aligned}
$$

and those associated with virtual transitions as

$$
\begin{aligned}
& \mathcal{K}_{Q}[\rho(t)] \\
& \quad=\left\{\sigma^{\dagger} \sigma,\left[\chi_{1}(t)+\chi_{2}(t) \sigma_{y}+\chi_{3}(t) \sigma^{\dagger} \sigma\right] \rho(t)\right\}+\text { H. c. }
\end{aligned}
$$

By defining the Fourier transformed correlation function $\gamma_{i}(\omega)=\int_{0}^{\infty} d \tau \Lambda_{i}(\tau) \exp (i \omega \tau)$, we may write the rate functions for real transitions as $\Gamma_{1}=\gamma_{x}(0)$, $\Gamma_{2}(t)=\left\{\gamma_{y}\left[\Omega_{r}(t)\right]+\gamma_{y}\left[-\Omega_{r}(t)\right]\right\} / 2$, and $\Gamma_{3}(t)=\left\{\gamma_{y}\left[\Omega_{r}(t)\right]\right.$ $\left.-\gamma_{y}\left[-\Omega_{r}(t)\right]\right\} / 2 i$. The rates associated with the virtual transitions are then $\chi_{1}(t)=\gamma_{z}(0) / 2-\left\{\gamma_{z}\left[\Omega_{r}(t)\right]+\right.$ $\left.\gamma_{z}\left[-\Omega_{r}(t)\right]\right\} / 4, \quad \chi_{2}(t)=\left\{\gamma_{z}\left[\Omega_{r}(t)\right]-\gamma_{z}\left[-\Omega_{r}(t)\right]\right\} / 4 i, \quad$ and $\chi_{3}(t)=\left\{\gamma_{z}\left[\Omega_{r}(t)\right]+\gamma_{z}\left[-\Omega_{r}(t)\right]\right\} / 2$.

As discussed in the main text, the Rabi oscillation measurements probe picosecond timescales. Virtual phonon processes, on the other hand, occur on a nanosecond timescale, and therefore the impact of the pulse on the virtual phonon dissipator is negligible. This allows us to replace the time-dependent dissipator $\mathcal{K}_{Q}[\rho(t)]$ with its time-independent counterpart, 
that is,

$$
\mathcal{K}_{Q}[\rho(t)] \approx \frac{\gamma_{\mathrm{pd}}}{2} \mathcal{L}_{\sigma^{\dagger} \sigma}[\rho(t)],
$$

where $\quad \mathcal{L}_{O}[\rho]=2 O \rho O^{\dagger}-\left\{O^{\dagger} O, \rho\right\}, \quad$ and $\quad \gamma_{\text {pd }}=$ $\int_{0}^{\infty} d \omega \mathcal{J}(\omega)^{2} n(\omega)[n(\omega)+1]$ is the pure-dephasing rate due to the virtual phonon scattering [47,49]. Using this expression, the final master equation becomes

$$
\begin{aligned}
\frac{\partial \rho(t)}{\partial t} \approx & -\frac{i \Omega_{r}(t)}{2}\left[\sigma_{x}, \rho(t)\right]+\mathcal{K}_{L}[\rho(t)] \\
& +\frac{\gamma_{p d}}{2} \mathcal{L}_{\sigma^{\dagger} \sigma}[\rho(t)] .
\end{aligned}
$$

By numerically solving Eq. (B4), we are able to explore how the final exciton population behaves as a function of pulse area and temperature. The final ingredient necessary to obtain a value proportional to the measured integrated intensity is the fraction of emission events into the zero phonon line. As shown in Ref. [24], this is simply the square of the thermal expectation of the lattice displacement operator, $B$. As such, for Fig. 3(b), we solve Eq. (B4) for the excited-state population and multiply by $B^{2}$.

\section{APPENDIX C: INDISTINGUISHABILITY CALCULATIONS}

Hong-Ou-Mandel measurements probe the coherence properties of photons on timescales relevant to the emitter lifetime, in this case, $T_{1} \sim 10^{3} \mathrm{ps}$. This is long after the pulse has finished, such that $\mathcal{K}_{L}[\rho(t)] \rightarrow 0$, meaning that the only phonon dephasing processes remaining in the master equation are virtual in nature; the master equation reduces to

$$
\frac{\partial \rho(t \gg \delta \tau)}{\partial t}=\frac{\gamma}{2} \mathcal{L}_{\sigma^{\dagger} \sigma}[\rho(t)]+\frac{\Gamma}{2} \mathcal{L}_{\sigma}[\rho(t)] .
$$

Here, $\gamma=\gamma_{\mathrm{pd}}+\tilde{\gamma}\left(\tau_{D}\right)$, where $\tilde{\gamma}\left(\tau_{D}\right)$ is introduced to capture dephasing associated with charge noise [27] and is given in Eq. (3).

If the phonon sideband has been removed from the QD spectrum [24], as is the case in our experiments, the indistinguishability can be found directly from the first-order correlation function $g^{(1)}(t, t+\tau)=\left\langle\sigma^{\dagger}(t+\tau) \sigma(t)\right\rangle$ via the expression $[25,26]$

$$
\mathcal{I}=\frac{\int_{0}^{\infty} d t \int_{0}^{\infty} d \tau\left|g^{(1)}(t, t+\tau)\right|^{2}}{\int_{0}^{\infty} d t \int_{0}^{\infty} d \tau g^{(1)}(t, t) g^{(1)}(t+\tau, t+\tau)} .
$$

By using the quantum regression theorem [53], we can express the correlation function in terms of the QD density operator $g^{(1)}(t, t+\tau)=\rho_{X X}(t) \exp [-(\Gamma+2 \gamma) \tau / 2]$, where $\rho_{X X}(t)=$ $\rho_{X X}(0) \exp (-\Gamma t / 2)$ is the time evolution of exciton population, with $\rho_{X X}(0)$ the excitonic population immediately following the excitation pulse. It is clear from the form of this correlation function that it is independent of the exciton coherence established by the pulse, and depends only on the exciton population. Thus, in situations where $T_{1} \gg \delta \tau$, the driving-dependent dephasing observed in the Rabi oscillations does not impact the indistinguishability. Furthermore, all occurrences of $\rho_{X X}(0)$ in Eq. (C2) will cancel, and we can say more generally that the indistinguishability is independent of the initial exciton state. Equations (C1) and (C2) lead to the indistinguishability expressions used in the main text.

When considering $p$-shell excitation of the $\mathrm{QD}$, we must account for the timing jitter introduced by the finite relaxation rate to the single-exciton $s$ shell from which photon emission occurs. To do so, we introduce a third "pump" state $|P\rangle$ [26] with energy $\Delta$, which decays to the $s$-shell state $|X\rangle$ with rate $\Gamma_{p \rightarrow s}$. The master equation describing this process, in addition to charge noise and virtual phonon scattering, is given by

$$
\begin{aligned}
\frac{\partial \rho(t)}{\partial t}= & -i[\Delta|P\rangle\langle P|, \rho(t)]+\frac{\gamma}{2} \mathcal{L}_{\sigma^{\dagger} \sigma}[\rho(t)] \\
& +\frac{\Gamma}{2} \mathcal{L}_{\sigma}[\rho(t)]+\frac{\Gamma_{p \rightarrow s}}{2} \mathcal{L}_{|X\rangle\langle P|}[\rho(t)] .
\end{aligned}
$$

Following the same procedure as before, though initializing the QD in $|P\rangle$, we obtain the modified expression for the indistinguishability $[25,26]$,

$$
\mathcal{I}=\left(\frac{\Gamma_{p \rightarrow s}}{\Gamma_{p \rightarrow s}+\Gamma}\right)\left(\frac{\Gamma}{\Gamma+2 \gamma}\right),
$$

where the timing jitter captured by the first factor acts to suppress the indistinguishability.

To determine the indistinguishability of the emitted single photons, we made use of an unbalanced free beam MZI. The contrast of the MZI has been ascertained by making use of a narrowband infrared diode laser whose wavelength is similar to the QD. The Michelson contrast $C_{M}$ has been measured by piezo-shifting the delay arm of the MZI to record the maximum and minimum of the laser power, $I_{\max }=(533.0 \pm$ 5.3) $\mu \mathrm{W}$ and $I_{\min }=(2.80 \pm 0.03) \mu \mathrm{W}$. By making use of the expression

$$
C_{M}=1-\epsilon=\frac{I_{\max }-I_{\min }}{I_{\max }+I_{\min }},
$$

we obtained a Michelson contrast of $C_{M}=\left(99.0_{-2.0}^{+1.0}\right) \%$. Additionally, the reflectivity $R$ and transmission $T$ of the imperfect 50:50 beam splitter were determined to be $R=0.485$ and $T=0.515$. We made use of the correction given by Santori et al. [16] to correct the area of the central peak of the coincidence histogram for parallel and orthogonal polarization according to the expressions

$$
\begin{gathered}
A_{\|} \propto\left(R^{3} T+R T^{3}\right)\left(1+2 g^{*}\right)-2(1-\epsilon)^{2} R^{2} T^{2} \nu, \\
A_{\perp} \propto\left(R^{3} T+R T^{3}\right)\left(1+2 g^{*}\right),
\end{gathered}
$$

where $g^{*}$ expresses the $g^{(2)}(0)$ value given by only taking into account the adjacent peaks at $\pm 12.2 \mathrm{~ns}$.
[1] J.-W. Pan, Z.-B. Chen, C.-Y. Lu, H. Weinfurter, A. Zeilinger, and M. Żukowski, Rev. Mod. Phys. 84, 777 (2012).

[2] P. Kok, W. J. Munro, K. Nemoto, T. C. Ralph, J. P. Dowling, and G. J. Milburn, Rev. Mod. Phys. 79, 135 (2007).

[3] J. L. O'Brien, Science 318, 1567 (2007).
[4] J. Nilsson, R. Stevenson, K. Chan, J. Skiba-Szymanska, M. Lucamarini, M. Ward, A. Bennett, C. Salter, I. Farrer, D. Ritchie et al., Nat. Photon. 7, 311 (2013).

[5] C. P. Dietrich, A. Fiore, M. G. Thompson, M. Kamp, and S. Höfling, Laser Photon. Rev. 10, 870 (2016). 
[6] J. McKeever, A. Boca, A. Boozer, R. Miller, J. Buck, A. Kuzmich, and H. Kimble, Science 303, 1992 (2004).

[7] F. Diedrich and H. Walther, Phys. Rev. Lett. 58, 203 (1987).

[8] T. Basché, W. E. Moerner, M. Orrit, and H. Talon, Phys. Rev. Lett. 69, 1516 (1992).

[9] R. Brouri, A. Beveratos, J.-P. Poizat, and P. Grangier, Opt. Lett. 25, 1294 (2000).

[10] C. Kurtsiefer, S. Mayer, P. Zarda, and H. Weinfurter, Phys. Rev. Lett. 85, 290 (2000).

[11] S. Castelletto, B. Johnson, V. Ivády, N. Stavrias, T. Umeda, A. Gali, and T. Ohshima, Nat. Mater. 13, 151 (2014).

[12] Y.-M. He, G. Clark, J. R. Schaibley, Y. He, M.-C. Chen, Y.-J. Wei, X. Ding, Q. Zhang, W. Yao, X. Xu et al., Nat. Nanotechnol. 10, 497 (2015).

[13] P. Tonndorf, R. Schmidt, R. Schneider, J. Kern, M. Buscema, G. A. Steele, A. Castellanos-Gomez, H. S. van der Zant, S. M. de Vasconcellos, and R. Bratschitsch, Optica 2, 347 (2015).

[14] I. Aharonovich, D. Englund, and M. Toth, Nat. Photon. 10, 631 (2016).

[15] P. Michler, A. Kiraz, C. Becher, W. Schoenfeld, P. Petroff, L. Zhang, E. Hu, and A. Imamoglu, Science 290, 2282 (2000).

[16] C. Santori, D. Fattal, J. Vuckovic, G. S. Solomon, and Y. Yamamoto, Nature (London) 419, 594 (2002).

[17] P. Lodahl, S. Mahmoodian, and S. Stobbe, Rev. Mod. Phys. 87, 347 (2015).

[18] Y.-M. He, O. Iff, N. Lundt, V. Baumann, S. Höfling, and C. Schneider, Nat. Commun. 7, 13409 (2016).

[19] O. Gazzano et al., Nat. Commun. 4, 1425 (2013).

[20] Y.-M. He, Y. He, Y.-J. Wei, D. Wu, M. Atature, C. Schneider, S. Höfling, M. Kamp, C.-Y. Lu, and J.-W. Pan, Nat. Nanotechnol. 8, 213 (2013).

[21] X. Ding, Y. He, Z. C. Duan, N. Gregersen, M. C. Chen, S. Unsleber, S. Maier, C. Schneider, M. Kamp, S. Höfling, C.-Y. Lu, and J.-W. Pan, Phys. Rev. Lett. 116, 020401 (2016).

[22] N. Somaschi, V. Giesz, L. De Santis, J. C. Loredo, M. P. Almeida, G. Hornecker, S. L. Portalupi, T. Grange, C. Anton, J. Demory, C. Gomez, I. Sagnes, N. D. L. Kimura, A. Lemaitre, A. Auffeves, A. G. White, L. Lanco, and P. Senellart, Nat. Photon. 10, 340 (2016).

[23] Y.-M. He, S. Maier, M. Emmerling, S. Gerhardt, M. Davanco, K. Srinivasan, C. Schneider, and S. Höfling, Optica 4, 802 (2017).

[24] J. Iles-Smith, D. P. S. McCutcheon, A. Nazir, and J. Mørk, Nat. Photon. 11, 521 (2017).

[25] P. Kaer, N. Gregersen, and J. Mork, New J. Phys. 15, 035027 (2013).

[26] S. Unsleber, D. P. S. McCutcheon, M. Dambach, M. Lermer, N. Gregersen, S. Höfling, J. Mørk, C. Schneider, and M. Kamp, Phys. Rev. B 91, 075413 (2015).

[27] A. Thoma, P. Schnauber, M. Gschrey, M. Seifried, J. Wolters, J.-H. Schulze, A. Strittmatter, S. Rodt, A. Carmele, A. Knorr, T. Heindel, and S. Reitzenstein, Phys. Rev. Lett. 116, 033601 (2016).

[28] P. Kaer, P. Lodahl, A.-P. Jauho, and J. Mørk, Phys. Rev. B 87, 081308 (2013).

[29] J. Houel, A. V. Kuhlmann, L. Greuter, F. Xue, M. Poggio, B. D. Gerardot, P. A. Dalgarno, A. Badolato, P. M. Petroff, A. Ludwig, D. Reuter, A. D. Wieck, and R. J. Warburton, Phys. Rev. Lett. 108, 107401 (2012).
[30] A. V. Kuhlmann, J. Houel, A. Ludwig, L. Greuter, D. Reuter, A. D. Wieck, M. Poggio, and R. J. Warburton, Nat. Phys. 9, 570 (2013).

[31] J. C. Loredo, N. A. Zakaria, N. Somaschi, C. Antón, L. De Santis, V. Giesz, T. Grange, M. A. Broome, O. Gazzano, G. Coppola, I. Sagnes, A. Lemaître, A. Auffèves, P. Senellart, M. P. Almeida, and A. G. White, Optica 3, 433 (2016).

[32] H. Wang, Z.-C. Duan, Y.-H. Li, S. Chen, J.-P. Li, Y.-M. He, M.-C. Chen, Y. He, X. Ding, C.-Z. Peng, C. Schneider, M. Kamp, S. Höfling, C.-Y. Lu, and J-W. Pan, Phys. Rev. Lett. 116, 213601 (2016).

[33] D. P. S. McCutcheon and A. Nazir, New J. Phys. 12, 113042 (2010).

[34] S. Unsleber, S. Maier, D. P. S. McCutcheon, Y.-M. He, M Dambach, M. Gschrey, N. Gregersen, J. Mørk, S. Reitzenstein, S. Höfling, C. Schneider, and M. Kamp, Optica 2, 1072 (2015).

[35] Y.-J. Wei, Y. He, Y.-M. He, C.-Y. Lu, J.-W. Pan, C. Schneider, M. Kamp, S. Höfling, D. P. S. McCutcheon, and A. Nazir, Phys. Rev. Lett. 113, 097401 (2014).

[36] A. J. Ramsay, T. M. Godden, S. J. Boyle, E. M. Gauger, A. Nazir, B. W. Lovett, A. M. Fox, and M. S. Skolnick, Phys. Rev. Lett. 105, 177402 (2010).

[37] D. P. S. McCutcheon and A. Nazir, Phys. Rev. Lett. 110, 217401 (2013).

[38] D. P. S. McCutcheon, Phys. Rev. A 93, 022119 (2016).

[39] J. Iles-Smith, D. P. S. McCutcheon, J. Mørk, and A. Nazir, Phys. Rev. B 95, 201305(R) (2017).

[40] E. A. Muljarov and R. Zimmermann, Phys. Rev. Lett. 93, 237401 (2004).

[41] T. Grange, N. Somaschi, C. Antón, L. De Santis, G. Coppola, V. Giesz, A. Lemaître, I. Sagnes, A. Auffèves and P. Senellart, Phys. Rev. Lett. 118, 253602 (2017).

[42] S. Maier, P. Gold, A. Forchel, N. Gregersen, J. Mørk, S. Höfling, C. Schneider, and M. Kamp, Opt. Express 22, 8136 (2014).

[43] A. J. Ramsay, A. V. Gopal, E. M. Gauger, A. Nazir, B. W. Lovett, A. M. Fox, and M. S. Skolnick, Phys. Rev. Lett. 104, 017402 (2010).

[44] A. Nazir and D. P. S. McCutcheon, J. Phys. Condens. Matter 28, 103002 (2016).

[45] K. Roy-Choudhury and S. Hughes, Opt. Lett. 40, 1838 (2015).

[46] A. Ulhaq, S. Weiler, C. Roy, S. M. Ulrich, M. Jetter, S. Hughes, and P. Michler, Opt. Express 21, 4382 (2013).

[47] A. Reigue, J. Iles-Smith, F. Lux, L. Monniello, M. Bernard, F. Margaillan, A. Lemaitre, A. Martinez, D. P. S. McCutcheon, J. Mørk, R. Hostein, and V. Voliotis, Phys. Rev. Lett. 118, 233602 (2017).

[48] Y.-J. Wei, Y.-M. He, M.-C. Chen, Y.-N. Hu, Y. He, D. Wu, C. Schneider, M. Kamp, S. Höfling, C.-Y. Lu, and J.-W. Pan, Nano Lett. 14, 6515 (2014).

[49] P. Tighineanu, C. L. Dreeßen, C. Flindt, P. Lodahl, and A. S. Sørensen, arXiv:1702.04812.

[50] T. Grange, R. Ferreira, and G. Bastard, Phys. Rev. B 76, 241304(R) (2007).

[51] R. Manson, K. Roy-Choudhury, and S. Hughes, Phys. Rev. B 93, 155423 (2016)

[52] H.-P. Breuer and F. Petruccione, The Theory of Open Quantum Systems (Oxford University Press on Demand, Oxford, 2002).

[53] H. Carmichael, Statistical Methods in Quantum Optics 1: Master Equations and Fokker-Planck Equations, Physics and Astronomy Online Library (Springer, New York, 1999). 